\title{
Publicación de resultados negativos
}

\author{
Publication of negative outcomes
}

\author{
Abilene C Escamilla Ortiz*
}

E stamos acostumbrados a leer en los artículos científicos resultados positivos, es raro ver publicados resultados negativos, ya que tiene más valor para los editores, lectores o casas editoriales, además de que es más fácil citarlos. ${ }^{1}$ Sobre todo está dado por el "publica o perece", donde el ganador es casi siempre por desempeño, por más artículos publicados o que estén en revistas de alto impacto. ${ }^{2}$

¿Qué son los resultados negativos? El estudio es pequeño y la muestra tiene poco poder estadístico, o los hallazgos no son concluyentes. Cuando a pesar de tener una buena muestra y un estudio bien planeado los resultados sugieren no tener efecto alguno. Cuando en vez del resultado esperado es todo lo contrario. ${ }^{1}$

¿Por qué los investigadores obtienen resultados negativos? La hipótesis original no era exacta y se basaba en suposiciones falsas e incorrectas. Debido a errores al no escoger el estudio adecuado, por no usar la prueba estadística apropiada para el estudio. Porque el investigador no pudo confirmar los hallazgos obtenidos en reportes preliminares. ${ }^{3}$

\section{¿POR QUÉ DIVULGAR RESULTADOS NEGATIVOS O INVÁLIDOS?}

La publicación de resultados negativos por un colega o por un grupo de colegas reconocidos puede provocar cambios en el propio estudio de investigación, por ejemplo, un medicamento no fue útil para cierto padecimiento, pero hacer el reporte ayuda a no exponer nuevamente a sujetos a los efectos de éste. ${ }^{3}$

\section{DECIDIRSE A PUBLICAR RESULTADOS NEGATIVOS}

Si el patrón de resultados es negativo, hay que revisar bien el método estadístico para corroborar que si lo es, el estudio debe repetirse varias veces para descartar fallas técnicas, buscar colaboración de ser posible con otros

${ }^{*}$ Editora, revista Cirujano General. orcid.org/00000001-5635-5845

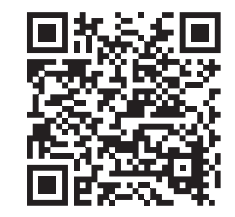
laboratorios e incrementar el rigor en la investigación. Si los resultados realmente son negativos y vale la pena reportarlos, se pueden publicar, ya que tendrán un impacto. ${ }^{3}$

La decisión de publicar resultados negativos no es fácil para autores o editores, se toman en consideración varias cosas, entre ellas, la cuestión

Citar como: Escamilla OAC. Publicación de resultados negativos. Cir Gen. 2020; 42 (4): 261-262. https://dx.doi. org/10.35366/101394 
ética por el uso de animales, sobre todo al tener que repetir un estudio. El resultado debe ser concluyente. ${ }^{3}$

\section{¿DÓNDE PUBLICAR RESULTADOS NEGATIVOS?}

Hay muchas formas de hacerlo, una de ellas es compartirlo con colegas publicándolo en el sitio web de la compañía o laboratorio, muy pocas revistas aceptan publicar resultados negativos, entre ellas están Positively Negative (PLOS One), Journal of Negative Results in Biomedicine, Journal of Negative Results- Ecology and Evolutionary Biology y Journal of Pharmaceutical Negative Results.

Publicar estudios clínicos controlados bien hechos puede evitar que otros colegas o investigadores gasten energía, tiempo y recursos en estudios similares, reduce el sesgo y ayuda de igual forma a no someter a participantes al mismo tratamiento. ${ }^{3}$

\section{¿QUÉ PODEMOS HACER PARA ENFRENTAR ESTE PROBLEMA EN LAS PUBLICACIONES BIOMÉDICAS?}

Estos estudios se pueden publicar en una sección separada de la revista donde se incluyan estos manuscritos previa revisión por pares, o en una sección con la metodología pre-estudio que describa cómo se llevó a cabo. Cuando el estudio esté concluido, la revista lo publicará completo, independientemente de los hallazgos, o lo incluirá en una sección de rechazados con la explicación de por qué fue rechazado. ${ }^{2}$

En la revista Cirujano General no hemos recibido este tipo de manuscritos, pero estamos abiertos a abrir una sección con esta clase de estudios, siempre y cuando tengan una buena metodología.

\section{REFERENCIAS}

1. Mlinaric A, Horvat M, Supak Smolcic V. Dealing with the positive publication bias: why you should really publish your negative results. Biochem Med. 2017; 27: 030201.

2. Sharma H, Verma $\mathrm{S}$. Is positive publication bias really a bias, or an intentionally created discrimination toward negative results? Saudi J Anaesth. 2019; 13: 352-355.

3. Bespalov A, Steckler T, Skolnick P. Be positive about negatives-recommendations for the publication of negative (or null) results. Eur Neuropsychopharmacol. 2019; 29: 1312-1320. 